\title{
ON A RELATED FUNCTION THEOREM
}

\author{
E. B. LEACH
}

1. In a previous note [1], the strong differential, a variant of the classical Fréchet differential, was defined. Strong differentiability at a point seems to be a good smoothness condition for related function theorems, being stronger than the insufficient condition of Fréchet differentiability at the point and weaker than Fréchet differentiability in a neighborhood of the point, together with continuity of the differential at the point.

In this note we state as a lemma a slight generalization of the theorem of [1]. Algebraic manipulation of the relations involved then enables us to extend the range over which the conclusion of the lemma is valid.

2. The following definition is given in [1].

Definition. Let $A$ and $B$ be open subsets of Banach spaces $U$ and $V$, respectively, and let $f: A \rightarrow B$ be a function. We say that $f$ has strong differential $\alpha$ at a point $x_{0} \in A$, if $\alpha: U \rightarrow V$ is a bounded linear transformation and for every $\epsilon>0$ there is a number $\delta>0$ such that

$$
\left|f\left(x^{\prime \prime}\right)-f\left(x^{\prime}\right)-\alpha\left(x^{\prime \prime}-x^{\prime}\right)\right| \leqq \epsilon\left|x^{\prime \prime}-x^{\prime}\right|,
$$

whenever $\left|x^{\prime}-x_{0}\right|<\delta$ and $\left|x^{\prime \prime}-x_{0}\right|<\delta$.

When $f$ has a strong differential $\alpha$ at $x_{0}$ we shall write $f^{\prime}\left(x_{0}\right)=\alpha$. The following lemma is the basic analytical tool of our discussion. (Throughout the rest of this note $f$ will denote a fixed function relating open subsets $A$ and $B$ of Banach spaces $U$ and $V$.)

Lemma. Let $\alpha: U \rightarrow V$ and $\beta: V \rightarrow U$ be bounded linear transformations such that $\beta \alpha \beta=\beta$. Let $x_{0} \in A$ and $y_{0} \in V$ satisfy $\beta\left(f\left(x_{0}\right)\right)=\beta\left(y_{0}\right)$ and $\beta\left(\alpha\left(x_{0}\right)\right)=x_{0}$. Finally, suppose that $f^{\prime}\left(x_{0}\right)=\alpha$. Then there are neighborhoods $A_{0}$ of $x_{0}$ and $B_{0}$ of $y_{0}$ (with $A_{0} \subset A$ ) such that:

(i) There is a unique function $g: B_{0} \rightarrow A_{0}$ satisfying $\beta(\alpha(g(y)))=g(y)$ and $\beta(f(g(y)))=\beta(y)$, for all $y \in B_{0}$.

(ii) $g$ is continuous, $g\left(y_{0}\right)=x_{0}$, and if for any $y_{1} \in B_{0}, f^{\prime}\left(g\left(y_{1}\right)\right)=\alpha_{1}$, then $g^{\prime}\left(y_{1}\right)=\gamma^{-1} \beta$, where $\gamma=1+\beta\left(\alpha_{1}-\alpha\right)$.

The neighborhoods $A_{0}$ and $B_{0}$ are described in terms of an $\epsilon>0$, chosen so that $\epsilon|\beta|<1 / 2$, and a $\delta>0$, chosen so that $\delta$ and $\epsilon$ satisfy the condition of differentiability of $f$ at $x_{0}$ and so that the sphere of 1962 .

Presented to the Society, February 23, 1963; received by the editors July 21, 
radius $\delta$ about $x_{0}$ is in $A$. Then $A_{0}$ is the sphere of radius $\delta$ about $x_{0}$ and $B_{0}$ is the sphere of radius $\delta / 2|\beta|$ about $y_{0}$. The function $g$ is the uniform limit of a sequence $\left\{g_{n}\right\}$ of functions defined recursively by:

$$
g_{0}(y)=x_{0} ; \quad g_{n+1}(y)=g_{n}(y)+\beta\left(y-f\left(g_{n}(y)\right)\right), \quad \text { if } n \geqq 0 .
$$

The entire proof corresponds exactly with the proof of the theorem of [1], and will be omitted.

Let $R$ be the relation consisting of pairs $(x, y)$, such that $x \in A$, $y \in V, \beta(\alpha(x))=x$ and $\beta(f(x))=\beta(y)$. The lemma asserts that near a point $\left(x_{0}, y_{0}\right)$ of $R$, points of $R$ consist exactly of the pairs $(g(y), y)$, provided $f^{\prime}\left(x_{0}\right)=\alpha$. The next theorem extends the range over which this conclusion is valid.

THEOREM. Let $\alpha: U \rightarrow V$ and $\beta: V \rightarrow U$ be bounded linear transformations such that $\beta \alpha \beta=\beta$. Let $x_{1} \in A$ and $y_{1} \in V$ be points satisfying $\beta\left(\alpha\left(x_{1}\right)\right)=x_{1}$ and $\beta\left(f\left(x_{1}\right)\right)=\beta\left(y_{1}\right)$. Finally, assume $f^{\prime}\left(x_{1}\right)=\alpha_{1}$, a transformation such that $\gamma=1+\beta\left(\alpha_{1}-\alpha\right)$ has a bounded inverse. Then there is a pair of neighborhoods $A_{1}$ of $x_{1}$ and $B_{1}$ of $y_{1}$ such that:

(i) There is a unique function $g: B_{1} \rightarrow A_{1}$ satisfying $\beta(\alpha(g(y)))=g(y)$ and $\beta(f(g(y)))=\beta(y)$, for all $y \in B_{1}$.

(ii) $g$ is continuous, $g\left(y_{1}\right)=x_{1}$ and if for any $y_{2} \in B_{1}, f^{\prime}\left(g\left(y_{2}\right)\right)=\alpha_{2}$, then $g^{\prime}\left(y_{2}\right)=\left[1+\beta\left(\alpha_{2}-\alpha\right)\right]^{-1} \beta$.

Proof. For any bounded linear transformation $\alpha_{2}: U \rightarrow V$, let $\beta_{1}=\gamma^{-1} \beta$ and $\gamma_{1}=1+\beta_{1}\left(\alpha_{2}-\alpha_{1}\right)$. Then $\gamma_{1}$ has a bounded inverse if and only if $\gamma \gamma_{1}$ has a bounded inverse. In this case, let $\beta_{2}=\gamma_{1}^{-1} \beta_{1}$. Then $\beta_{2}=\gamma_{1}^{-1} \gamma^{-1} \beta=\left(\gamma \gamma_{1}\right)^{-1} \beta$, where $\gamma \gamma_{1}=\gamma\left[1+\gamma^{-1} \beta\left(\alpha_{2}-\alpha_{1}\right)\right]=\gamma$ $+\beta\left(\alpha_{2}-\alpha_{1}\right)=1+\beta\left(\alpha_{1}-\alpha\right)+\beta\left(\alpha_{2}-\alpha_{1}\right)=1+\beta\left(\alpha_{2}-\alpha\right)$. In particular, if $\alpha_{2}=\alpha, \beta_{2}=\beta$, and so the pairs $(\alpha, \beta)$ and $\left(\alpha_{1}, \beta_{1}\right)$ are symmetrically related.

Further, an identity of the type we are considering involving $\alpha$ and $\beta$ may be replaced by the corresponding identity about $\alpha_{1}$ and $\beta_{1}$. To see this, first note that because $\beta \alpha \beta=\beta$, then $\beta \alpha \gamma=\beta \alpha+\beta \alpha \beta\left(\alpha_{1}-\alpha\right)$ $=\beta \alpha_{1}$, and composing with $\gamma^{-1}$ on the right we obtain:

$$
\beta \alpha=\beta \alpha_{1} \gamma^{-1} \text {. }
$$

Also, $\gamma \beta=\beta+\beta\left(\alpha_{1}-\alpha\right) \beta=\beta \alpha_{1} \beta$, and composing with $\gamma^{-1}$ on the left we obtain :

$$
\beta=\gamma^{-1} \beta \alpha_{1} \beta .
$$

Now $\beta_{1} \alpha_{1} \beta_{1}=\gamma^{-1} \beta \alpha_{1} \gamma^{-1} \beta=\gamma^{-1} \beta \alpha \beta=\gamma^{-1} \beta=\beta_{1}$, using (3) at the second step. For any $x$, if $\beta(\alpha(x))=x$, then $\beta_{1}\left(\alpha_{1}(x)\right)=\gamma^{-1}\left(\beta\left(\alpha_{1}(x)\right)\right)$ $=\gamma^{-1}\left(\beta\left(\alpha_{1}(\beta(\alpha(x)))\right)\right)=\beta(\alpha(x))=x$, using (4) at the third step. Fi- 
nally, if $\beta(f(x))=\beta(y)$, for a pair $(x, y)$, then $\beta_{1}(f(x))=\gamma^{-1}(\beta(f(x)))$ $=\gamma^{-1}(\beta(y))=\beta_{1}(y)$. In view of the symmetric relation between $(\alpha, \beta)$ and $\left(\alpha_{1}, \beta_{1}\right)$, the converse propositions are also true: if $\beta_{1} \alpha_{1} \beta_{1}=\beta_{1}$, then $\beta \alpha \beta=\beta$, etc. It can also be shown similarly that $\beta$ is a left or right inverse to $\alpha$ if and only if the same relation holds between $\beta_{1}$ and $\alpha_{1}$.

From these considerations the relation $R$ is equivalent to the relation $R_{1}$, in which $\alpha$ and $\beta$ are replaced by $\alpha_{1}$ and $\beta_{1}$. Since $f^{\prime}\left(x_{1}\right)=\alpha_{1}$, the conditions of the lemma are valid, and there is a function $g: B_{1} \rightarrow A_{1}$ such that the pairs $(g(y), y)$ are the points of $R_{1}$, and so of $R$, near $\left(x_{1}, y_{1}\right)$. For the point $y_{2}$, of (ii), the lemma asserts $g^{\prime}\left(y_{2}\right)$ $=\gamma_{1}^{-1} \beta_{1}$, where $\gamma_{1}=1+\beta\left(\alpha_{2}-\alpha_{1}\right)$. But we have already shown that $\gamma_{1}^{-1} \beta_{1}=\left[1+\beta\left(\alpha_{2}-\alpha\right)\right]^{-1} \beta$. This proves the theorem.

3. Other relations. In [1], the relations $g f \beta=\beta, g \alpha \beta=g$, and $g f g=g$ were discussed as local identities under restrictive conditions on the reference points. We can discuss them more generally in the light of the related function theorem above.

For any $y$, if $\beta(y) \in A$, then $(\beta(y), f(\beta(y))) \in R$, by easy calculation. If $f^{\prime}\left(\beta\left(y_{0}\right)\right)=\alpha_{1}$, for one such $y_{0}$ and $1+\beta\left(\alpha_{1}-\alpha\right)$ has a bounded inverse, then the theorem gives a unique $g: B_{1} \rightarrow A_{1}$, where $A_{1}$ is a neighborhood of $\beta\left(y_{0}\right)$ and $B_{1}$ is a neighborhood of $f\left(\beta\left(y_{0}\right)\right)$, such that the pairs $(g(y), y)$ are in $R$. But for $y$ near $y_{0}$, the pairs $(\beta(y), f(\beta(y)))$ are points of $R$ near $\left(\beta\left(y_{0}\right), f\left(\beta\left(y_{0}\right)\right)\right)$, so by uniqueness of $g, \beta(y)$ $=g(f(\beta(y)))$.

Similarly, if $(x, y) \in R$, then $(x, \alpha(\beta(y))) \in R$. If $\left(x_{1}, y_{1}\right) \in R$ and $f^{\prime}\left(x_{1}\right)=\alpha_{1}$, satisfying the condition of the theorem, the theorem gives functions $g$ and $\bar{g}$ giving rise to pairs in $R$ near the pairs $\left(x_{1}, y_{1}\right)$ and $\left(x_{1}, \alpha\left(\beta\left(y_{1}\right)\right)\right)$, respectively. Now if $y$ is near $y_{1},(g(y), y) \in R$, so $(g(y), \alpha(\beta(y))) \in R$, and by uniqueness of $\bar{g}, g(y)=\bar{g}(\alpha(\beta(y)))$, for $y$ near $y_{1}$.

Finally, if $(x, y) \in R$, then $(x, f(x)) \in R$. For such a point $\left(x_{1}, y_{1}\right)$, if $f^{\prime}\left(x_{1}\right)=\alpha_{1}$ is suitably well behaved, functions $g$ and $\bar{g}$ exist determining points of $R$ near $\left(x_{1}, y_{1}\right)$ and $\left(x_{1}, f\left(x_{1}\right)\right)$, respectively. For $y$ near $y_{1},(g(y), y) \in R$, so $(g(y), f(g(y))) \in R$, the latter point being near to $\left(x_{1}, f\left(x_{1}\right)\right)$, and so by uniqueness of $\bar{g}, g(y)=\bar{g}(f(g(y)))$, for $y$ near $y_{1}$.

\section{REFERENCE}

1. E. B. Leach, $A$ note on inverse function theorems, Proc. Amer. Math. Soc. 12 (1961), 694-697.

\section{Case Institute of Technology}

